\title{
Influence of the Timing of Percutaneous Coronary Intervention on Clinical Outcomes in Non-ST-Elevation Myocardial Infarction
}

\author{
Kamuran Tekin, MD1, Caglar Emre Cagliyan, MD², Ibrahim Halil Tanboga, MD², Mehmet Balli, MD², \\ Onur Kadir Uysal, MD², Bugra Ozkan, MD², Osman Ziya Arik, MD², and Murat Cayli, MD² \\ ${ }^{1}$ Department of Cardiology, Batman State Hospital, Batman, Turkey \\ ${ }^{2}$ Department of Cardiology, Adanana Numune Education and Research Hospital, Istanbul, Turkey
}

Background and Objectives: We have intended to investigate the influence of the timing of invasive procedures on all-cause mortality, recurrent myocardial infarction (MI), re-hospitalization due to cardiac causes and left ventricular function over a 3-month period among patients with Non-ST-elevation myocardial infarction (NSTEMI).

Subjects and Methods: A total of 131 NSTEMI patients with moderate-high Thrombolysis in Myocardial Infarction risk scores, who had been admitted to our department between July 2011-December 2011 were included in our study. They had been randomized into 2 groups according to the timing of the percutaneous coronary intervention (PCI). Patient undergoing PCl in the first 24 hours of hospitalization were named the "Early Invasive Group" and those undergoing PCl between 24-72 hours of hospitalization were named the "Delayed Invasive Group". All patients were followed up for 3 months.

Results: Third month left ventricular ejection fraction (LVEF) values were higher in the early invasive group (59.9 $\pm 6.0 \%$ vs. $54.1 \pm 8.7 \%$; $p<0.001)$. Recurrent MI rates were lower in the early invasive group ( $2.9 \%$ vs. $14.5 \% ; p=0.016$ ). Similarly, hospitalization rates due to cardiac events were lower in the early invasive group ( $8.7 \%$ vs. 30.6\%; $p=0.001)$. All cause mortality appeared to be lower in the early invasive group, although not to a statistically significant degree ( $0 \%$ vs. $4.8 \% ; p=0.065)$.

Conclusion: The early invasive strategy appears to be more effective for the reduction of recurrent Ml, re-hospitalization due to cardiac events, and the preservation of 3rd month LVEF in patients with moderate-high risk NSTEMI when compared to a delayed invasive strategy.

(Korean Circ J 2013;43:725-730)

KEY WORDS: Percutaneous coronary intervention; Myocardial infarction; Prognosis.

\section{Introduction}

Non-ST-elevation myocardial infarction (NSTEMI), which is the most common referral form of acute coronary syndrome (ACS), represents the majority of patients that undergo percutaneous coro-

Received: April 15, 2013

Revision Received: August 19, 2013

Accepted: September 10, 2013

Correspondence: Kamuran Tekin, MD, Department of Cardiology, Batman State Hospital, Batman 72010, Turkey

Tel: 90-5533966203, Fax: 90-3223387017

E-mail: kamurantekin@gmail.com

- The authors have no financial conflicts of interest.

This is an Open Access article distributed under the terms of the Creative Commons Attribution Non-Commercial License (http://creativecommons. org/licenses/by-nc/3.0) which permits unrestricted non-commercial use, distribution, and reproduction in any medium, provided the original work is properly cited. nary intervention (PCI). Among NSTEMI patients, the essential goals of coronary revascularization are minimizing angina symptoms, maintaining the systolic functions of the ventricles by resolving myocardial ischemia, lessening repetitive myocardial infarction (MI), and improving the prognosis in the short and mid-term by decreasing the progression to death. In randomized controlled studies (RCS), it has been found that invasive procedures in the treatment of moderate to high risk NSTEMI is superior in offering protection against unwanted cardiac events compared to conservative treatment options. $^{12)}$ There were many RCS performed in order to determine the optimal timing of coronary angiography and revascularization in NSTEMI patients. The results of a meta-analysis that include four of these studies revealed that, although early catheterization performed on the first day of hospitalization is a reliable and superior method in preventing repetitive myocardial ischemia and decreasing the amount of hospitalization time required, it did not result in a significant difference in mortality rates. ${ }^{3)}$ This issue of optimal in- 
vasive timing remains unresolved through RCSs. Further and more extensive studies are required to determine a strategy to decrease the mortality rates resulting from coronary artery disease. The aim of revascularization is to minimize the symptoms and hospitalization periods, and improve the short and long-term prognosis of patients. As a result, the therapeutic strategy that will be chosen in NSTEMI patients in the acute period affects early, mid-term and longterm prognosis in a significant way.

\section{Subjects and Methods}

A total of 139 NSTEMI patients with moderate to high Thrombolysis in Myocardial Infarction (TIMI) risk scores that were being followed up at the Adana Numune Research and Education Hospital Cardiology Department between July 2011 and December 2011 were enrolled in the study. They had been randomized into 2 groups according to the timing of $\mathrm{PCl}$ through computer-generated randomization. Patients undergoing PCl in the first 24 hours of hospitalization were named the "Early Invasive Group", and those undergoing $\mathrm{PCl}$ between 24-72 hours of hospitalization were named the "Delayed Invasive Group". All patients were followed up for 3 months. Of these 139 patients, eight were excluded due to inadequacy of in-hospital data and the nonexistence of follow up records. The study was initiated with 131 moderate- to high-risk NSTEMI (85 male, 46 female, and an average age of $56.9 \pm 10.2$ year-old) patients. Those patients with a TIMI risk score of $<3$, a history of coronary artery bypass graft operation, bypass decision following coronary angiography, severe ventricular arrhythmia, symptoms of heart failure and a left ventricle ejection fraction (LVEF) <35\%, hemodynamic instability, conditions such as continuing or recurring ischemia that necessitate emergency $\mathrm{PCl}$, and those patients who refused $\mathrm{PCl}$ and refused to sign the consent forms, as well as those who were pregnant were excluded from this study.

\section{Echocardiographic evaluation}

All echocardiographic measurements were taken when patients were at complete rest and at the left lateral decubiti's position. Along with routine echocardiographic evaluations, left ventricle end systolic volumes and end diastolic volumes and ejection fractions were measured using the "Simpson" method (152). An echocardiographic evaluation was performed at discharge and at the $3 \mathrm{rd}$ month during a clinic visit by a cardiologist who was unaware of the study purposes.

\section{Clinical follow up and results}

Our study group was clinically followed up for a total of a 3-month period. During this time, clinic visits were performed in the $1 s t, 2 n d$, and 3rd months. At the end of our study, the timing and frequency of undesired cardiovascular events were recorded. Repetitive MI, hospitalization due to cardiac reasons, and death due to any reason were regarded as major adverse cardiac events. Repetitive MI was regarded to be present when there was an elevation of cardiac markers during the post hospitalization period, along with chest pain relevant to ischemia or ischemic electrocardiography (ECG) changes.

\section{Statistical analysis}

Statistical analysis was conducted using Statistical Package for the Social Sciences (SPSS) 14.0 software (SPSS Inc., Chicago, IL, USA). Numerical variables were indicated as mean \pm standard deviation or median, and categorical variables as percentages or rates. In order to test the differences between the groups, a Student t-test was used for numerical variables that had regular distribution, and the Mann-Whitney $U$ test was employed if there was an irregular distribution. Categorical variables were analyzed by the chi-square test and Fisher's exact test. Kaplan Meier survival analysis was used in the analysis of endpoints that occurred after the follow up period, and a log rank test was performed to test the differences. A p of $<0.05$ was regarded as statistically significant.

\section{Results}

There were 69 patients in the early invasive group and 62 in the delayed invasive group. Basal demographic and clinical features (age, gender and risk factors) of the patients, ECG irregularities, and TIMI risk scores were similar (Table 1). Infarct related artery distribution was similar in both groups. Moreover, there were mostly 3 vessel diseases in both groups. In none of the patients was the left main coronary artery the responsible lesion. The number of stents used was found to be higher in patients in the delayed invasive group (1.42 \pm 0.70 vs. $1.71 \pm 0.88, p=0.04)$. Drug eluting stent (DES) use was particularly low in both groups and the DES and bare metal stent distribution was similar $(p=0.207)$. Between the two therapy groups, the rate of obtaining TIMI-III flow tended to be higher in the post$\mathrm{PCl}$ early invasive therapy group ( $97.1 \%$ vs. 88.7\%, $p=0.059$ ) (Table 2). As expected, the hospitalization period in the early invasive group was markedly lower than that in the delayed invasive group (33.6 \pm 5.9 hours vs. $81.4 \pm 38.3$ hours, and $p<0.001$ ). Moreover, brain natriuretic peptide (BNP) levels were distinctly higher in the delayed invasive therapy group (75.4 \pm 124.2 vs. $163.1 \pm 249.8, p<0.001)$. There were no significant differences between the LVEF and C-reactive protein (CRP) levels of the two groups, as measured at the time of discharge (Table 3). In the third month of LVEF measurements, it was determined that the levels were better preserved in the early invasive therapy group when compared to the delayed invasive thera- 
py group (59.3 $\pm 6.0 \%$ vs. $54.1 \pm 8.7 \%, \mathrm{p}<0.001)$. Repetitive MI between the therapy strategies were found to be significantly lower in the early invasive therapy group when compared to the delayed invasive group (2.9\% and $14.5 \%$, respectively, $p=0.016$ ). Similarly, cardiac related hospitalization rates were significantly lower in the early invasive group compared to those in the delayed invasive gr- oup (8.7\% vs. $30.6 \%$, respectively, $p=0.001)$. Although there was no death in the early invasive group, there were three deaths in the delayed invasive group. Two of the three deaths were caused by ACS and one was caused by acute decompensated chronic heart failure. Deaths related to any reason were determined to be lower in the early invasive group than in the delayed invasive group (0\% vs.

Table 1. Demographic and basal clinical features in early invasive and delayed invasive groups

\begin{tabular}{lccc}
\hline Variables & Early invasive $(\mathbf{n = 6 9 )}$ & Delayed invasive $(\mathbf{n = 6 2 )}$ & $\mathbf{p}$ \\
\hline Age (year) & $58.1 \pm 10.3$ & $55.6 \pm 10.1$ & \\
Gender (\%) & & 0.169 \\
$\quad$ Male & 59.4 & 71.2 & \\
$\quad$ Female & 40.6 & 28.8 & \\
Diabetes (\%) & 31.9 & 45.2 & \\
Hypertension (\%) & 55.1 & 50.2 & 0.168 \\
Hyperlipidemia (\%) & 62.3 & 50.1 & 0.120 \\
Smoking (\%) & 60.9 & 48.4 & 0.157 \\
ECG abnormalities (\%) & 81.2 & 80.6 & 0.153 \\
TIMl risk score (average) & $4.10 \pm 0.85$ & $4.19 \pm 1.02$ & 0.941
\end{tabular}

ECG: electrocardiography, ECG abnormalities: ST-segments changes and T wave abnormalities, TIMI: Thrombolysis in Myocardial Infarction

Table 2. Distributions of the coronary angiography data between the therapy groups of early invasive and delayed invasive

\begin{tabular}{lcc}
\hline Variables & Early invasive $(\mathbf{n = 6 9 )}$ & Delayed invasive (n=62) \\
\hline IRA (\%) & & $0(0.0)$ \\
\hline LMCA & $0(0.0)$ & $34(54.8)$ \\
\hline LAD & $38(55.1)$ & $16(25.8)$ \\
CX & $20(29.0)$ & $12(19.4)$ \\
\hline RCA & $11(15.9)$ & $11(17.7)$ \\
Prevalence of CAD (\%) & & $16(25.8)$ \\
\hline One vessel & $9(13.0)$ & $35(56.5)$ \\
Two vessels & $40(58.1)$ & $1.71 \pm 0.88$ \\
Three vessels & $40(58.1)$ & 0.739 \\
Number of stents used & $1.42 \pm 0.70$ & $55(88.7)$ \\
Stent type (\%) & & $7(11.3)$ \\
BMS & $65(94.2)$ & 88.7 \\
\hline DES & $4(5.8)$ & 0.040 \\
\hline Post-procedure TIMI III flow (\%) & 97.1 & 0.207 \\
\hline
\end{tabular}

IRA: infarct related artery, LMCA: left main coronary artery, LAD: left anterior descending artery, Cx: left circumflex artery, RCA: right coronary artery, CAD: coronary artery disease, BMS: bare metal stent, DES: drug eluting stent, TIMI: Thrombolysis in Myocardial Infarction

Table 3. Distribution of discharge, laboratory, and echocardiographic data and hospitalization periods between early invasive and delayed invasive therapy groups

\begin{tabular}{lccc}
\hline Variables & Early invasive $(\mathbf{n = 6 9 )}$ & Delayed invasive $(\mathbf{n = 6 2 )}$ & $\mathbf{p}$ \\
\hline BNP $(\mathrm{pg} / \mathrm{dL})$ & $75.4 \pm 124.2$ & $163.1 \pm 249.8$ & 0.001 \\
CRP $(\mathrm{mg} / \mathrm{dL})$ & $3.4 \pm 3.7$ & $2.7 \pm 2.1$ & 0.877 \\
LVEF-discharge (\%) & $56.5 \pm 7.5$ & $55.6 \pm 7.3$ & 0.660 \\
Hospitalization period (hour) & $33.6 \pm 5.9$ & $81.4 \pm 38.3$ & $<0.001$ \\
\hline
\end{tabular}

CRP: C-reactive protein, BNP: brain natriuretic peptide, LVEF: left ventricular ejection fraction 
Table 4. Prevalence of death occurring for any kind of reason, cardiac related hospitalization, recurring myocardial infarction in 3 months, and third month LVEF between the therapeutic strategies

\begin{tabular}{|c|c|c|c|}
\hline Variables & Early invasive $(n=69)$ & Delayed invasive $(n=62)$ & p \\
\hline LVEF-3rd month (\%) & $59.3 \pm 6.0$ & $54.1 \pm 8.7$ & $<0.001$ \\
\hline Recurring infarction (\%) & $2(2.9)$ & $9(14.5)$ & 0.016 \\
\hline Recurring hospitalization (\%) & $6(8.7)$ & 19 (30.6) & 0.001 \\
\hline Death occurring for any kind of reason (\%) & - & $3(4.8)$ & 0.065 \\
\hline Compound endpoints $\left.{ }^{*} \%\right)$ & $6(8.7)$ & $19(30.6)$ & 0.001 \\
\hline
\end{tabular}

*Recurring infarction, cardiac related hospitalization and death related to any kind of reason have been defined as the compound endpoints. LVEF: left ventricular ejection fraction

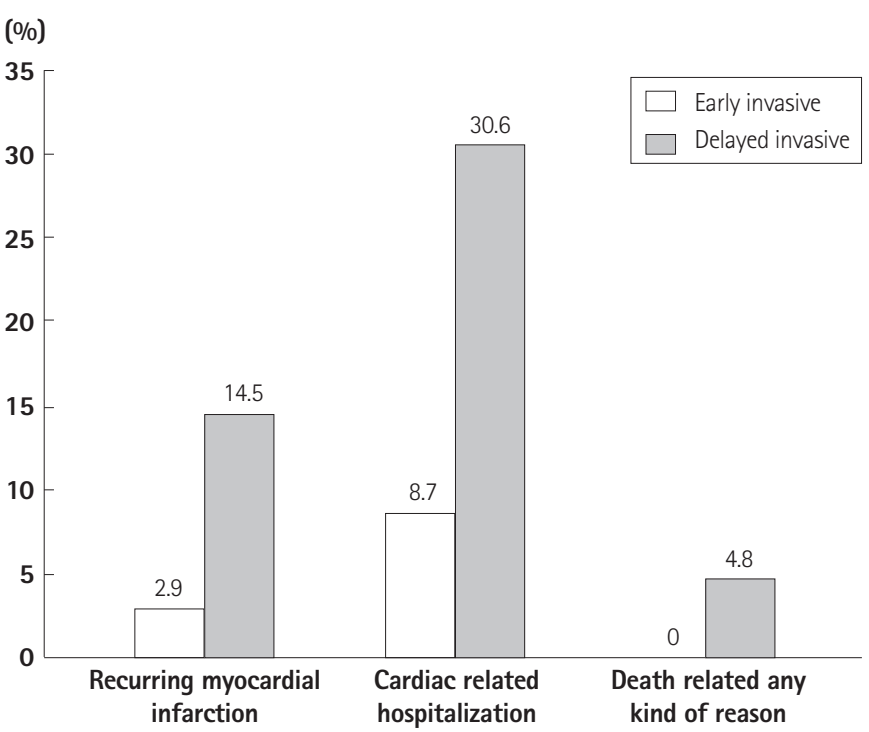

Fig. 1. Recurring myocardial infarction, cardiac related hospitalization, and death prevalence related to any reason within the first three month period between different treatment strategies.

$4.8 \%$, respectively, $\mathrm{p}=0.065$ ). Compound endpoints were also significantly lower in the early invasive therapy group (Table 4, Fig. 1).

\section{Discussion}

It was determined in our study that with the early invasive therapy of NSTEMI patients of moderate- to high-risk, there was much more TIMI-III flow obtained, a lower hospitalization duration, a better preserved left ventricle function, a decrease in the compound endpoint results, including recurring $\mathrm{MI}$ and cardiac related hospitalization, and a tendency toward a decrease of mortality due to any reason.

ST-elevated myocardial infarction (STEMI), NSTEMI, and unstable angina pectoris (UAP) are similar in their etiologies in the sense that they all have in common a pathological atherosclerotic plaque rupture history. Although the pathophysiology of these settings known as ACS is similar, their clinical courses differ. In UAP, in-hospital mortality is low and its 1-year mortality was found to be 1.6\%-a figure close to that of chronic stabile angina." The highest in-hospital mortality rate is 7\% among STEMI patients, but their mortality rates are equal with those of NSTEMI patients at the 6th month. ${ }^{56)}$ In long-term follow-ups, it has been shown that the mortality rate among NSTEMI patients is two times that of STEMI patients in the fourth year. ${ }^{7)}$ The actual reason behind this difference in mortality is that NSTEMI patients tended to be older and have a higher rate of comorbid conditions, such as diabetes and chronic renal failure.

\section{The importance of invasive therapy in non-ST-elevation myocardial infarction treatment}

With the development of new therapy strategies, multiple treatment options are now available for NSTEMI patients. However, mortality and morbidity rates in this group remain high. Among these therapy options, there were two therapy strategies that came to the forefront in clinical settings. One of them was the early invasive therapy (in the first 72 hours following referral) or routine coronary angiography and $\mathrm{PCl}$ to the proper lesion. The second is conservative or selective invasive therapy option. In the second option, stabilization is expected by therapy, and invasive therapy is performed in cases of ongoing or recurrent angina. There are many randomized control studies relating to planning an ideal acute therapy regimen, the timing of interventional procedures, and long-term therapeutic strategies. In a meta-analysis performed of data obtained from FRISC II, RITA 3, and Invasive Versus Conservative Treatment in Unstable Coronary Syndromes (ICTUS), it was found that patients benefited more from interventional therapy when compared to conservative therapy. ${ }^{122}$ In subgroup analyses performed at the end of the studies conducted independently of the used risk scores, it was shown that high-risk group patients benefited more from invasive therapy. The aim of revascularization is to minimize the symptoms and hospitalization period, and improve the short- and long-term prognosis of patients. As a result, the therapeutic strategy that will be chosen in NSTEMI patients in the acute period affects early, midterm and long-term prognosis significantly. That is why European Society of Cardiology guidelines recommend an invasive therapy strategy for ACS patients in moderate and high-risk groups. ${ }^{8}$ 


\section{Percutaneous coronary intervention timing in Non-ST elevation myocardial infarction}

Although an early invasive diagnostic approach is indicated in the diagnosis and planning of sequential therapy, the timing of a routine invasive procedure remains controversial. Despite many RCTs and meta-analysis on the issue, the optimal intervention time in NSTEMI patients remains undefinitive. In the Intracoronary Stenting with Antithrombotic Regimen Cooling-Off (ISAR-COOL) study, in which the optimal intervention timing was assessed among 401 randomized NSTEMI patients, it was found that death and the MI rate were both significantly lower in the patient group in whom the intervention was performed in the first 6 hours. ${ }^{9}$ In a more recent and more expanded study (of 7749 patients) of the Acute Catheterization and Urgent Intervention Triage (ACUITY) strategy, patients were divided into three groups according to the intervention time ( $<8$ hours, 8-24 hours, and $>24$ hours), and they were followed up for a year. Results from the study showed that the $<8$ hours and 8-24 hour groups had similar death and death or MI and compound ischemia rates, whereas all three endpoints in the $>24$ hour group were significantly higher than those in the other groups. ${ }^{10)}$ We have divided the moderate-high-risk NSTEMI patients into two groups depending on the PCl timing-first 24 hours and 24-72 hours. After a three-month follow up, we have determined that recurring $\mathrm{MI}$ and cardiac related hospitalization was significantly less, LVEF was preserved better, and the rate of obtaining a TIMI-III flow following $\mathrm{PCl}$ was higher in patients on whom the $\mathrm{PCl}$ was performed in the first 24 hours.

In our study, when patient groups were compared in regards to cardiac related hospitalizations, the rate was found to be significantly lower in the early invasive group (8.7\% vs. 30.6\%). It was seen that the risk of hospitalization due to cardiac causes was higher in patients with a diabetes mellitus history, low LVEF during discharge, high levels of BNP and CRP at discharge, low coronary artery TIMI flow following PCl, and those with high TIMI risk scores. Similarly, in the ICTUS study in which early interventional therapy was compared with traditional therapy, it was reported that angina related re-hospitalization rates were lower with early invasive therapy. ${ }^{11)}$

In our study, when patient groups were compared with regards to recurring $\mathrm{MI}$, the rate was found to be significantly lower in the early invasive group (2.9\% vs. $14.5 \%$ ). In a meta-analysis of three trials-Angioplasty to Blunt the Rise of Troponin in Acute Coronary Syndromes Randomized for an Immediate or Delayed Intervention (ABOARD), Early or Late Intervention in unStable Angina, ISAR-COOL, and TIMACS-early catheterization followed by coronary intervention on the first day of hospitalization was shown to be safe and superior in terms of presenting a lower risk of recurrent ischaemia and promising a shorter hospital stay.
In our study, when patient groups were compared with regards to compound endpoints (recurring MI, cardiac related hospitalization, and death due to any reason), it was observed that the endpoints were significantly lower in the early invasive therapy group. In the OPTIMA, ABOARD, and TIMACS studies, there were no differences in the compound endpoints with different strategies. These results do not correlate with our findings. However, in the ACUITY study, the largest scale study conducted so far, it was determined that compound endpoints were lower in the early invasive therapy group. ${ }^{10) 12-14)}$

\section{Clinical relevance}

It has been shown that it is possible to have a decrease in the mid-term MUCE rate in NSTEMI patients, who represent the most common referral groups of ACS patients and the most $\mathrm{PCl}$ performed groups, with appropriate invasive therapy timing. Along with this benefit, it can be deduced that, especially in centers where catheterization rates are high, early invasive therapy will lead to a decrease in hospitalization periods for NSTEMI patients and will, therefore, result in more efficient health service delivery. As resource use, such as drugs, nurses, physicians, and accessory personnel decreases, significant health care cost cutting can occur. However, for the cost effectiveness analysis of this treatment strategy, further analysis focusing on the economic benefits of these findings is required.

\section{Conclusion}

Early invasive therapy strategy in moderate- to high-risk NSTEMI patients is more effective in decreasing recurrent myocardium infarction and cardiac related recurring hospitalization and preserving the left ventricle functions compared to delayed invasive therapy strategy.

\section{Limitations}

The most important limitation of our study is the low number of patients in the groups randomized for therapy strategies. Moreover, the fact that follow up durations were not long enough may affect the frequencies of endpoints, thus the results of the study.

\section{References}

1. Mehta SR, Cannon CP, Fox KA, et al. Routine vs selective invasive strategies in patients with acute coronary syndromes: a collaborative metaanalysis of randomized trials. JAMA 2005;293:2908-17.

2. Patel TN, Bavry AA, Kumbhani DJ, Ellis SG. A meta-analysis of randomized trials of rescue percutaneous coronary intervention after failed fibrinolysis. Am J Cardiol 2006;97:1685-90.

3. Katritsis DG, Siontis $G C$, Kastrati $A$, et al. Optimal timing of coronary 
angiography and potential intervention in non-ST-elevation acute coronary syndromes. Eur Heart J 2011;32:32-40.

4. Bigi R, Cortigiani L, Colombo P, Desideri A, Bax JJ, Parodi O. Prognostic and clinical correlates of angiographically diffuse non-obstructive coronary lesions. Heart 2003;89:1009-13.

5. Savonitto $S$, Ardissino D, Granger $C B$, et al. Prognostic value of the admission electrocardiogram in acute coronary syndromes. JAMA 1999; 281:707-13.

6. Volmink JA, Newton JN, Hicks NR, Sleight P, Fowler GH, Neil HA. Coronary event and case fatality rates in an English population: results of the Oxford myocardial infarction incidence study. The Oxford Myocardial Infarction Incidence Study Group. Heart 1998;80:40-4.

7. Terkelsen CJ, Lassen JF, Nørgaard BL, et al. Mortality rates in patients with ST-elevation vs. non-ST-elevation acute myocardial infarction: observations from an unselected cohort. Eur Heart J 2005;26:18-26.

8. Hamm CW, Bassand JP, Agewall S, et al. ESC Guidelines for the management of acute coronary syndromes in patients presenting without persistent ST-segment elevation: The Task Force for the management of acute coronary syndromes (ACS) in patients presenting without persistent ST-segment elevation of the European Society of Cardiology (ESC). Eur Heart J 2011;32:2999-3054.
9. Neumann FJ, Kastrati A, Pogatsa-Murray G, et al. Evaluation of prolonged antithrombotic pretreatment ("cooling-off" strategy) before intervention in patients with unstable coronary syndromes: a randomized controlled trial. JAMA 2003;290:1593-9.

10. Sorajja $P$, Gersh BJ, Cox DA, et al. Impact of delay to angioplasty in patients with acute coronary syndromes undergoing invasive management: analysis from the ACUITY (Acute Catheterization and Urgent Intervention Triage strategY) trial. J Am Coll Cardio/ 2010;55:1416-24.

11. de Winter RJ, Windhausen $F_{1}$ Cornel $J H_{1}$ et al. Early invasive versus selectively invasive management for acute coronary syndromes. N Engl J Med 2005;353:1095-104.

12. Mehta $S R$, Granger $C B$, Boden WE, et al. Early versus delayed invasive intervention in acute coronary syndromes. N Engl J Med 2009;360: 2165-75.

13. Riezebos RK, Ronner E, Ter Bals E, et al. Immediate versus deferred coronary angioplasty in non-ST-segment elevation acute coronary syndromes. Heart 2009;95:807-12.

14. Montalescot G, Cayla G, Collet JP, et al. Immediate vs delayed intervention for acute coronary syndromes: a randomized clinical trial. JAMA 2009;302:947-54. 